\title{
ASSESSMENT OF NURSING CARE AND SOME RELATED FACTORS ON PATIENTS WITH TONSILLECTOMY IN AN GIANG EYE - EAR NOSE THROAT - DENTAL HOSPITAL
}

\author{
Huynh Trong Nguyen ${ }^{1}$, Nguyen Dinh Phuc ${ }^{2}$ \\ ${ }^{1}$ An Giang Eye - Ear Nose Throat - Dental Hospital; ${ }^{2}$ Thang Long Univesity
}

\begin{abstract}
Objectives: To identify nursing care and some related factors on patients with tonsillectomy in An Giang eye - ear nose throat - dental hospital. Method: A crosssectional study was conducted on 157 patients with tonsillectomy. Results: The nursing follow-up activities are well evaluated; in which the pain monitoring rate is best evaluated (91.7\%); followed by monitoring cough/bleeding (87.3\%); monitoring communication problems $86.6 \%$. The nursing instructions to the patient about the problems after surgery is also appreciated quite well. Including, instruction for resting (93\%), instruction for dental hygiene (90.5\%), instruction for speaking (89.8\%); instruction for adherence to drug treatment (89.6\%); instructions for adherence to the diet (75.2\%); personal hygiene/ exercise instructions (71.3\%). $89.8 \%$ of the patients rated the general care results of the nurses as good. The study results also showed that ethnicity and pain level after surgery are related to the results of care for patients with tonsillectomy. Patients with Viet ethnicity rate better care outcomes OR=3.82 (95\% Cl: 1.17-12.49); The more pain the patient has after surgery, the better the care: moderate/mild pain OR=3.29 (95\%Cl: 1.05-10.27); severe pain/very severe pain $O R=7.14$ (95\% Cl: 1.04-320.07). Conclusion: It is necessary to pay more attention to health counseling and guidance for Hoa, Khmer ethnicity patients, and patient who have pain after surgery.
\end{abstract}

Keywords: Tonsillectomy, nursing care, postoperative care.

\section{INTRODUCTION}

Tonsillitis is a common disease in bacterial infections in the ear, nose and throat. Chronic tonsillitis often recurs with episodes of acute inflammation several times a year. This pathology not only affects the quality of life, but is also quite dangerous. Because tonsillitis can cause many local complications such as abscess, inflammation; and neighboring complications such as laryngitis, sinusitis, ear infection, or distant complications such as heart, kidney, and joint [1]. Tonsillectomy is the surgical removal of the entire palatal tonsil block [2]. There are many surgical methods of tonsillectomy from classical to modern such as Sluder surgery, dissection, electric knife cutting, laser, coblator, plasma knife. These surgical methods have various advantages in terms of reducing complications, shortening surgery time,
Cor. author: Huynh Trong Nguyen

Address: An Giang Eye-Ear Nose Throat-Dental Hospital

Email: tnguyen.tlu@gmail.com
Received: Jan 15, 2022

Accepted: Mar 01, 2022

Published: Mar 02, 2022 
recovery time and especially reducing pain levels during and after surgery. However, after tonsillectomy surgery, there are still many potential complications such as: pain, bleeding, fever, infection... and anxiety of the patient. Therefore, the role of the nurse is very important in supporting the patient. Nurses need to advise patients and their families before surgery, monitor and care for patients after surgery with medication regimens, psychological nutrition. These nursing care will help to normalize the postoperative period and the patient to return to normal life soon. Therefore, we carry out this study with the goal of analyzing the results of care for tonsillectomy patients at An Giang eye - ear nose throat - dental hospital and some related factors.

\section{RESEARCH SUBJECTS AND} METHODS

\subsection{Research subjects}

The patient underwent tonsillectomy with a Coblator knife at An Giang eye - ear nose throat - dental hospital

\section{- Inclusion criteria}

The patient has complete preoperative laboratory indicators and clearly recorded medical records.

The patient and family agreed to participated in the study.

\section{- Exclusion criteria}

Patients with medical diseases: decompensated heart failure, cirrhosis of the liver, bronchial asthma, blood clotting disorders...;

Currently circulating respiratory infections in the locality;

Female patient in menstruating period.
Patients did not cooperate with the study or did not follow up adequately.

\subsection{Research period and setting}

Research period: from November, 2020 to April, 2021.

Research setting: An Giang eye - ear nose throat - dental hospital

2.3. Study design: A cross-sectional study

\subsection{Sample size and sampling} method:

- Sample size: apply the formula to calculate sample size for proportion estimate:

$$
n=Z_{1-\alpha / 2}^{2} \frac{p(1-p)}{d^{2}}
$$

n: sample size;

$\alpha=0,05$ 1,962).

$Z_{(1-\alpha / 2)}$ at confidence level $95 \%\left(Z_{(1-\alpha / 2)}=\right.$

$p=88,4 \%$ (According to the results of good patient care after tonsillectomy, Ninh Thi Khuyen, 2011) [3].

$\mathrm{d}$ : Allowable deviation between the rate obtained from the study sample and the rate of the population. Deviation $5 \%, d=0.05$.

$$
=>\mathrm{n}=157 \text {. }
$$

- Sampling method: Convenience sampling, all subjects met the criteria during the research period from November 1, 2020 to April 30, 2021.

2.5. Research variables, evaluation criteria

- Demographic factors: age, gender, occupation, education qualification, place of residence, ethnicity. 
- Clinical characteristics before surgery: common clinical symptoms, level of tonsil hypertrophy (based on medical records)

- Postoperative characteristics: bleeding (early, late), pain 48 hours after surgery (based on medical records combined with interviews)

Evaluation of nursing care after tonsillectomy in the study was using 5 points Likert scale [4], [3]:

+ No counseling $=0$ points (M1).

+ Having counseling but patients do not understand $=1$ point (M2).

+ Having counseling, patients understand but cannot follow $=2$ points (M3).

+ Having counseling, patients understand and follow $=3$ points (M4).

+ Having counseling, patients understand and follows well $=4$ points (M5).

- Each consulting content was evaluated

+ Good: the patient answered M4 and M5 correctly.

+ Not good: Reached M3 and M2 and M1 consulting content.

- Monitoring activities of nurses (4 contents)

- Instructional activities of nurses (7 contents)

- Patient satisfaction: divided into 5 levels (Level $1=$ very dissatisfied, level 2 $=$ unsatisfied, level $3=$ normal, level $4=$ satisfied, level $5=$ very satisfied).

- Results of nursing care activities on patients

+ Good level: $80 \%$ of nursing care activities and patients are satisfied with nursing care.
+ Fair level: Reaching less than $80 \%$ of nursing care activities, patients are not satisfied.

- Factors associated with the outcome of tonsillectomy care: demographics, pain level...

\subsection{Data analysis}

The data were synthesized by using SPSS 16.0 software.

Descriptive statistical including mean, percentage, standard deviation were used to describe variables of study.

Analysis of factors related to the outcome of care by using OR $(95 \% \mathrm{Cl})$, the relationship has statistical significance $p<0.05$.

\subsection{Research ethics}

The research proposal was approved by the Ethical Review Board of Thang Long University Council the Scientific Council of An Giang eye - ear nose throat - dental hospital. An informed consent was obtained to ensure that the subjects voluntarily participated in this study. the women participating in the study were provided with full information about the study including the purpose, research methods and rights when participating in the study. Participants have the right to withdraw from the study at any time. 


\section{RESULTS}

Table 1. Demographic factors of the subjects

\begin{tabular}{|llcc|}
\hline & Characteristics & Frequency & $\%$ \\
\hline \multirow{3}{*}{ Gender } & Male & 79 & 50.3 \\
& Female & 78 & 49.7 \\
\hline \multirow{3}{*}{ Age } & $\leq 15$ & 88 & 56.1 \\
& $16-30$ & 34 & 21.7 \\
& $>30$ & 35 & 22.3 \\
\hline \multirow{3}{*}{ Education qualification } & Elementary & 87 & 55.4 \\
& Secondary School, High School & 29 & 18.5 \\
& Intermediate, diploma & 23 & 14.7 \\
& Undergraduate, graduate & 18 & 11.5 \\
\hline \multirow{3}{*}{ Occupation } & Student & 97 & 61.8 \\
& Workers and Employees & 32 & 20.4 \\
& Housewife, freelance & 28 & 17.8 \\
\hline \multirow{3}{*}{ Ethnicity } & Viet & 137 & 87.3 \\
& Hoa & 13 & 8.3 \\
& Khmer & 7 & 4.4 \\
\hline
\end{tabular}

Research subjects are male and female with similar proportions $(50.3 \%$ and $49.7 \%)$. More than half of the study subjects were under the age of $15(56.1 \%)$. Nearly threequarters of the study subjects had less than high school education (73.8\%). Research subjects are students account for the largest percentage (61.8\%).

Table 2. Characteristics clinical

\begin{tabular}{|c|c|c|c|}
\hline \multicolumn{2}{|c|}{ Characteristics } & \multirow{2}{*}{$\begin{array}{c}\text { Frequency } \\
111\end{array}$} & \multirow{2}{*}{$\begin{array}{c}\% \\
70.7\end{array}$} \\
\hline \multirow{4}{*}{$\begin{array}{l}\text { Common clinical } \\
\text { symptoms }\end{array}$} & Difficulty swallowing & & \\
\hline & Snoring while sleeping & 73 & 46.5 \\
\hline & Stop breathing while sleeping & 27 & 17.2 \\
\hline & Bad smelling breath & 96 & 61.1 \\
\hline \multirow{4}{*}{$\begin{array}{l}\text { The level of tonsil } \\
\text { hypertrophy }\end{array}$} & Level I & 43 & 27.4 \\
\hline & Level II & 36 & 22.9 \\
\hline & Level III & 51 & 32.5 \\
\hline & Level IV & 27 & 17.2 \\
\hline & Total & 157 & 100 \\
\hline
\end{tabular}


The most common clinical symptom is difficulty swallowing $(70.7 \%)$. Subjects with level III tonsil hypertrophy accounted for the highest rate $(32.5 \%)$

Table 3. Characteristics of bleeding and pain after surgery

\begin{tabular}{|c|c|c|c|}
\hline \multicolumn{2}{|c|}{ Characteristics } & \multirow{2}{*}{$\begin{array}{c}\text { Frequency } \\
130\end{array}$} & \multirow{2}{*}{$\begin{array}{c}\% \\
82.8\end{array}$} \\
\hline \multirow{5}{*}{ Bleeding } & No & & \\
\hline & $<6$ hours & 4 & 2.6 \\
\hline & $6-24$ hours & 9 & 5.7 \\
\hline & $>24-48$ hours & 8 & 5.1 \\
\hline & $>48$ hours & 6 & 3.8 \\
\hline \multirow{6}{*}{$\begin{array}{l}\text { Pain after surgery } 48 \\
\text { hours }\end{array}$} & Worst possible pain & 3 & 1.9 \\
\hline & Very severe pain & 5 & 3.2 \\
\hline & Severe pain & 23 & 14.7 \\
\hline & Moderate pain & 31 & 19.8 \\
\hline & Mild pain & 43 & 27.4 \\
\hline & No pain & 52 & 33.1 \\
\hline & Total & 157 & 100 \\
\hline
\end{tabular}

Most of the subjects did not bleed after surgery (82.8\%); There were $66.9 \%$ of patients with pain after surgery, in which the rate of mild pain accounted for the highest rate $(27.4 \%)$.

Table 4. Nursing care after surgery $(n=157)$

\begin{tabular}{|lcccc|}
\hline \multicolumn{1}{|c}{ Nursing care } & \multicolumn{2}{c}{ Good } & \multicolumn{3}{c|}{ Not goof } \\
& Frequency & $\%$ & Frequency & $\%$ \\
\hline Monitoring activies & & & & \\
Monitor phlegm & 136 & 86.6 & 21 & 13.4 \\
Monitor cough & 137 & 87.3 & 20 & 12.7 \\
Monitor bleeding & 137 & 87.3 & 20 & 12.7 \\
Monitor pain & 144 & 91.7 & 13 & 8.3 \\
\hline Instruction activities & & & & \\
Speaking & 141 & 89.8 & 16 & 10.2 \\
Dental hygiene & 142 & 90.5 & 15 & 9.5 \\
Resting & 146 & 93.0 & 11 & 7.0 \\
Adherence to the diet & 118 & 75.2 & 39 & 24.8 \\
Medication adherence & 125 & 89.6 & 32 & 20.4 \\
Personal hygiene & 112 & 71.3 & 45 & 28.7 \\
Exercise & 112 & 71.3 & 45 & 28.7 \\
\hline Satisfied with the quality of nursing care & 141 & 89.8 & 16 & 10.2 \\
\hline
\end{tabular}


Most of the subjects assessed that the nurses performed well the content of postoperative follow-up. In which, pain monitoring was best evaluated $(91.7 \%)$.

After surgery, the nurses performed instruction activities for the patient such as speaking, dental hygiene, adherence to medication, diet, and exercise. Instructing patients to rest, and instructing dental hygiene were highly rated at over $90 \%(93.0 \% ; 90.5 \%)$. The rate of speaking instruction activities is $89.8 \%$; guidelines for adherence to drug treatment $89.6 \%$; compliance with the $75.2 \%$ diet; the percentage of patients who rated it well in personal hygiene/exercise activities was $71.3 \%$.

The majority of subjects were satisfied with the quality of nursing care $(89.8 \%)$

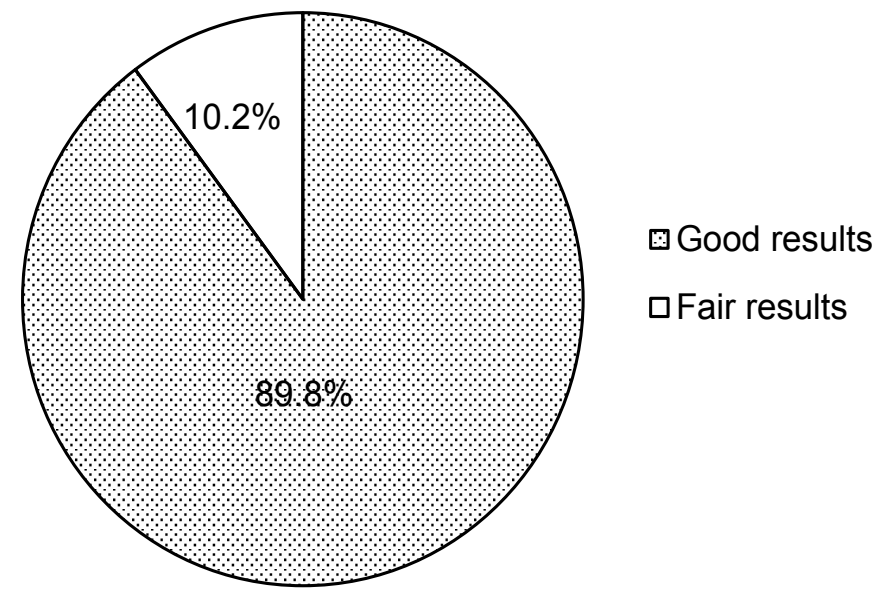

Figure 1. Overall care performance assessment

$89.8 \%$ of the subjects rated the general care results of nurses at goog level.

Table 5. Some factors related to care outcomes

\begin{tabular}{|llcccc|}
\hline \multirow{2}{*}{ Factors } & \multicolumn{2}{c}{ Care outcomes } & & \multirow{2}{*}{ OR } \\
& & Good & Not & $\mathbf{p}_{\text {sig }}$ & (95\%Cl) \\
\hline \multirow{2}{*}{ Ethnicity } & Hoa/Khmer & 15 & 5 & & 1 \\
& Viet & 126 & 11 & 0.03 & $3.82(1.17-12.49)$ \\
\hline \multirow{3}{*}{ Pain level } & No pain & 42 & 10 & & 1 \\
& Mild pain/ Moderate pain & 69 & 5 & 0.03 & $3.29(1.05-10.27)$ \\
& Severe pain/ Very severe pain & 30 & 1 & 0.03 & $7.14(1.04-320.07)$ \\
\hline$\quad$ Total & $\mathbf{1 4 1}$ & $\mathbf{1 6}$ & & \\
\hline
\end{tabular}

Ethnicity and pain level after surgery are related to the outcome of patient care after tonsillectomy $(p<0.05)$. Gender, age, occupation, education qualification, operative time, blood loss, postoperative bleeding, and fever were not related to patient outcome.

Patients with Viet ethnicity rate better care outcomes OR=3.82 (95\% Cl: 1.17-12.49); The more pain the patient has after surgery, the better the care: moderate/mild pain $\mathrm{OR}=3.29(95 \% \mathrm{Cl}: 1.05-10.27)$; severe pain/very severe pain $\mathrm{OR}=7.14(95 \% \mathrm{Cl}: 1.04-$ $320.07)(p<0.05)$. 


\section{DISCUSSION}

\subsection{Clinical characteristics}

The most common clinical symptoms were difficulty swallowing (70.7\%); Bad smelling breath (61.1\%); snoring while sleeping $(46.5 \%)$; Stop breathing while sleeping (17.2\%). According to the research results of Pham Tran Anh (2016), other functional symptoms such as itchy throat, difficulty swallowing were also found in over $70 \%$ of patients [5]. Swallowing problems is a very valuable symptom suggestive of acute inflammation, overproduction of tonsils, this rate in our study was $70.7 \%$. According to the study of Duong Huu Nghi and Nguyen Tan Dinh, the rate of swallowing problems was high 89.0, throat itching 43.0\% [6]. Most patients with tonsillitis experience bad smelling breath despite good oral hygiene. Due to repeated inflammation of the tonsils, white pus-filled cavities around the tonsils, throat mucosa and even in the nasopharynx appear. The amount of leftover food that is stagnate in the deep cavities inside the tonsils is not removed, decomposed by bacteria and causes this condition. According to the research results of Duong Huu Nghi, the rate of patients with bad smelling breath was $27 \%$ [6]. The study results showed that the percentage of subjects with the highest degree of tonsil hypertrophy level III was $32.5 \%$; Levels I and II account for lower rates of $27.4 \%$ and $22.9 \%$. According to the research results of Dinh Van Luan (2020), the tonsils with level III and IV overstimulation accounted for $83.4 \%$ and the overactive level II accounted for $16.2 \%$ [7].

\subsection{Outcomes of patient care and some related factors}

In order to have the best care for the patient before, during and after surgery, the patients were explained before surgery about the progression of the disease after surgery, instructions on living and eating regimen, especially lifestyle right after surgery, time for re-examination. Every day, nurses ask, encourage and evaluate the patient's process performance. After tonsillectomy, the patient should be closely monitored for the wound to heal quickly to limit complications. In our study, most of the subjects assessed good the monitoring activities of nurses; in which pain monitoring was best rated $91.7 \%$; monitoring cough/ bleeding $87.3 \%$; monitoring activities had $86.6 \%$ of the subjects rated good. Bleeding after surgery is not uncommon and it can persist for up to two weeks afterward. The nurse instructs the patient to lie on his or her side, with no headrest and face to one side. Do not spit, do not swallow saliva, let saliva come out onto absorbent paper placed under the corner of the mouth. After surgery, the nurse guides the patient to practice speaking, dental hygiene, adherence to medication, diet, and exercise. Nurses guided patients to rest, oral hygiene was rated as good, over 90\% (93.0\%; 90.5\%). The rate of speaking instruction activities is $89.8 \%$; instruction for adherence to drug treatment $89.6 \%$; the percentage of patients who rated it well in personal hygiene/exercise activities was $71.3 \%$. After tonsillectomy, the patients will have many difficulties in eating, so it is necessary to have a diet according to the instructions of the nurse. However, the activity of guiding patients to adhere to the nurse's diet was not as highly appreciated as other activities ( $25 \%$ of patients rated the nursing's dietary guidance as not good). This result can be explained because nurses have not really 
paid attention to this issue during patient care. The percentage of subjects who rated the general care results of nurses as good was $89.8 \%$; There are $10.2 \%$ of bad reviews. The results of Nguyen Tuan's study showed that the majority of patients after surgery evaluated the nursing care well $(88.4 \%)$ [8].

Ethnicity and pain level after surgery are related to the outcome of patient care after tonsillectomy $(p<0.05)$. Gender, age, occupation, education qualification, operative time, blood loss, postoperative bleeding, and fever were not related to patient outcome. Patients with Viet ethnicity rate better care outcomes $\mathrm{OR}=3.82$ (95\% Cl: 1.17-12.49). It means that patients from the Kinh ethnicity group received good care 3.82 times higher than that of the Hoa/ Khmer ethnicity group. Health insurance policy in recent years has prioritized supporting ethnic minorities in An Giang. The State pays health insurance premiums and enjoys $100 \%$ of medical examination and treatment costs under health insurance for ethnic minorities in difficult and extremely difficult areas. Therefore, the fact that ethnic minorities have access to public health facilities is of concern, however, it is appropriate for medical staff to pay much attention to support during treatment and surgery at the hospital.

Postoperative pain is one of the problems patients face after tonsillectomy. Pain after surgery causes a very uncomfortable feeling that affects the psychology of the patient, slows down the recovery process after surgery, especially increases the risk of becoming chronic pain. Our study showed the more pain the patient has after surgery, the better the care: moderate/mild pain OR=3.29 (95\%Cl: 1.05-10.27); severe pain/very severe pain $\mathrm{OR}=7.14(95 \% \mathrm{Cl}$ : 1.04-320.07) $(p<0.05)$., the relationship is statistically significant with $p<0.05$. During treatment, pain symptoms will often be common and immediately reflected by the patient. Therefore, it was often urgent for nurses to pay attention and care for these patients.

\section{CONCLUSION}

The nursing follow-up activities are well evaluated; in which the pain monitoring rate is best evaluated $(91.7 \%)$; followed by monitoring cough/bleeding (87.3\%); monitoring communication problems $86.6 \%$. The nursing instructions to the patient about the problems after surgery is also appreciated quite well. Including, instruction for resting (93\%), instruction for dental hygiene $(90.5 \%)$, instruction for speaking (89.8\%); instruction for adherence to drug treatment (89.6\%); instructions for adherence to the diet $(75.2 \%)$; personal hygiene/exercise instructions (71.3\%). $89.8 \%$ of the patients rated the general care results of the nurses as good. The study results also showed that ethnicity and pain level after surgery are related to the results of care for patients with tonsillectomy. Patients with Viet ethnicity rate better care outcomes $\mathrm{OR}=3.82(95 \% \mathrm{Cl}$ : 1.17 12.49); The more pain the patient has after surgery, the better the care: moderate/mild pain $\mathrm{OR}=3.29$ (95\%Cl: 1.05-10.27); severe pain/very severe pain $\mathrm{OR}=7.14(95 \% \mathrm{Cl}$ : 1.04-320.07). It is necessary to pay more attention to health counseling and guidance for Hoa, Khmer ethnicity patients, and patient who have pain after surgery. 


\section{REFERENCES}

1. Nguyen Dinh Bang (2005), Lectures Ear, nose and throat, Medicine Publishing House, 74-81.

2. Ronald Alastair McNeill (1960), "A history of tonsillectomy: two millenia of trauma, haemorrhage and controversy", The Ulster medical journal, 29(1), p. 59. https://pubmed.ncbi.nlm.nih.gov/20476427/

3. Ninh Thi Khuyen et.al (2011), “ Take care of patient after tonsillectomy ", The internal journal of the $12^{\text {th }}$ National Otolaryngology Conference, p. 321-326.

4. Nguyen Thuy Doan Trang (2009), "Develop a care plan for patients after tonsillectomy ", The internal journal of the Otolaryngology, 1, p. 562.

5. Pham Tran Anh and Pham Thi Bich Dao (2016), "Clinical and paraclinical characteristics of patients with acute purulent tonsillitis at the outpatient
Department, National Otolaryngology Hospital of Vietnam", Journal of Preventive Medicine, XXVI (188), p. 223-228.

6. Duong Huu Nghi, Nguyen Tan Dinh and Nguyen Thi To Trinh (2007), Survey on improvement of functional symptoms of patients over 15 years old after tonsillectomy at Can Tho Otolaryngology Hospital from June 2007 to November 2007, Proceedings of scientific topics of the Global Otolaryngology Conference national year 2009, An Giang, p. 250-257.

7. Dinh Van Luan and Cao Minh Thanh (2020), " Study on clinical characteristics and ratio of Biofilm on tonsils of patients undergoing tonsillectomy ", Jounal of Vietnam Otolaryngology, 65 - 49.

8. Nguyen Tuan, Ninh Thi Khuyen and Ngo Thi Xuan (2011), " Take care of patient after tonsillectomy ", Medical Journal of Ho Chi Minh City, 15, p. 126-232. 